First publ. in: Appetite 52 (2009), 2, pp. 513-516

\title{
Vegetarianism and food perception. Selective visual attention to meat pictures
}

\author{
Jessica Stockburger $^{\mathrm{a}, *}$, Britta Renner ${ }^{\mathrm{a}}$, Almut I. Weike ${ }^{\mathrm{b}}$, Alfons O. Hamm ${ }^{\mathrm{b}}$, Harald T. Schupp ${ }^{\mathrm{a}}$ \\ a Department of Psychology, University of Konstanz, 78457 Konstanz, Germany \\ ${ }^{\mathrm{b}}$ Department of Psychology, University of Greifswald, 17487 Greifswald, Germany
}

\section{Introduction}

The domain of food is a primary source of affect. Powerful mechanisms shape affective responding towards food stimuli according to nutritional needs of the organism (e.g., sweet preference, taste aversion; Rozin \& Vollmecke, 1986). However, in humans, ideational reasoning provide further means determin ing affective responding, potentially overriding the pleasure based on the flavor of selected food items (Martins \& Pliner, 2005; Rozin, Markwith, \& Stoess, 1997). For instance, meat products may elicit a strong dislike and even disgust due to moral (e.g., linking meat consumption to cruelty, environmental degradation and political concerns) and health aspects (Allen, Wilson, Ng, \& Dunne, 2000; Amato \& Partridge, 1989; Fessler, Arguello, Mekdara, \& Macias, 2003; Kenyon \& Barker, 1998). Thus, vegetarianism provides a model system to explore the effects of a hedonic shift from liking to dislike towards specific food items based on ideational reasoning.

The affect system has pervasive effects on various psychological processes. The present study focused on the affective guidance of selective attention processes. Such a mechanism assures that significant stimuli automatically gain focused attention facilitating appropriate approach or avoidance behaviors (Lang, Bradley, \&

\footnotetext{
* Corresponding author at: Institute of Psychology, University of Konstanz, PO Box 36, 78457 Konstanz, Germany.

E-mail address: jessica.stockburger@uni-konstanz.de (J. Stockburger).
}

Cuthbert, 1997; Öhman, Flykt, \& Lundqvist, 2000). Here, using a passive viewing task, we examined whether vegetarians' negative affect towards meat turns corresponding visual stimuli into efficient attention catchers. Brain potentials enable one to measure the attention capture of affective stimuli at the level of initial stimulus perception. Previous studies revealed that the late positive potential (LPP), which occurs between 300 and $700 \mathrm{~ms}$ after stimulus onset, is enlarged when viewing unpleasant (e.g., mutilations, threat) and pleasant (erotic scenes, adventures) compared to neutral (household objects, neutral faces) pictures (Cuthbert, Schupp, Bradley, Birbaumer, \& Lang, 2000; Schupp, Flaisch, Stockburger, \& Junghöfer, 2006). Accordingly, the heigh tened affective salience of meat stimuli in vegetarians was expected to increase selective attention processes as indexed by the LPP. Furthermore, this effect was predicted to be specific to meat (i.e. not apparent for comparable non meat pictures), because meat is the distinct food category rejected by vegetarians. Previous research suggests that enlarged LPP amplitudes to significant stimuli reflect an implicit process (Ito \& Cacioppo, 2000). In order to explore this issue, we included a condition in which participants performed an explicit attention task in which target stimuli were defined by an additional food category (desserts). It was hypothesized that vegetarians would show enhanced LPP amplitudes to meat pictures even when both meat and vegetable pictures represent non target stimuli, thus support ing the idea that enlarged LPP amplitudes reflect an implicit process. 

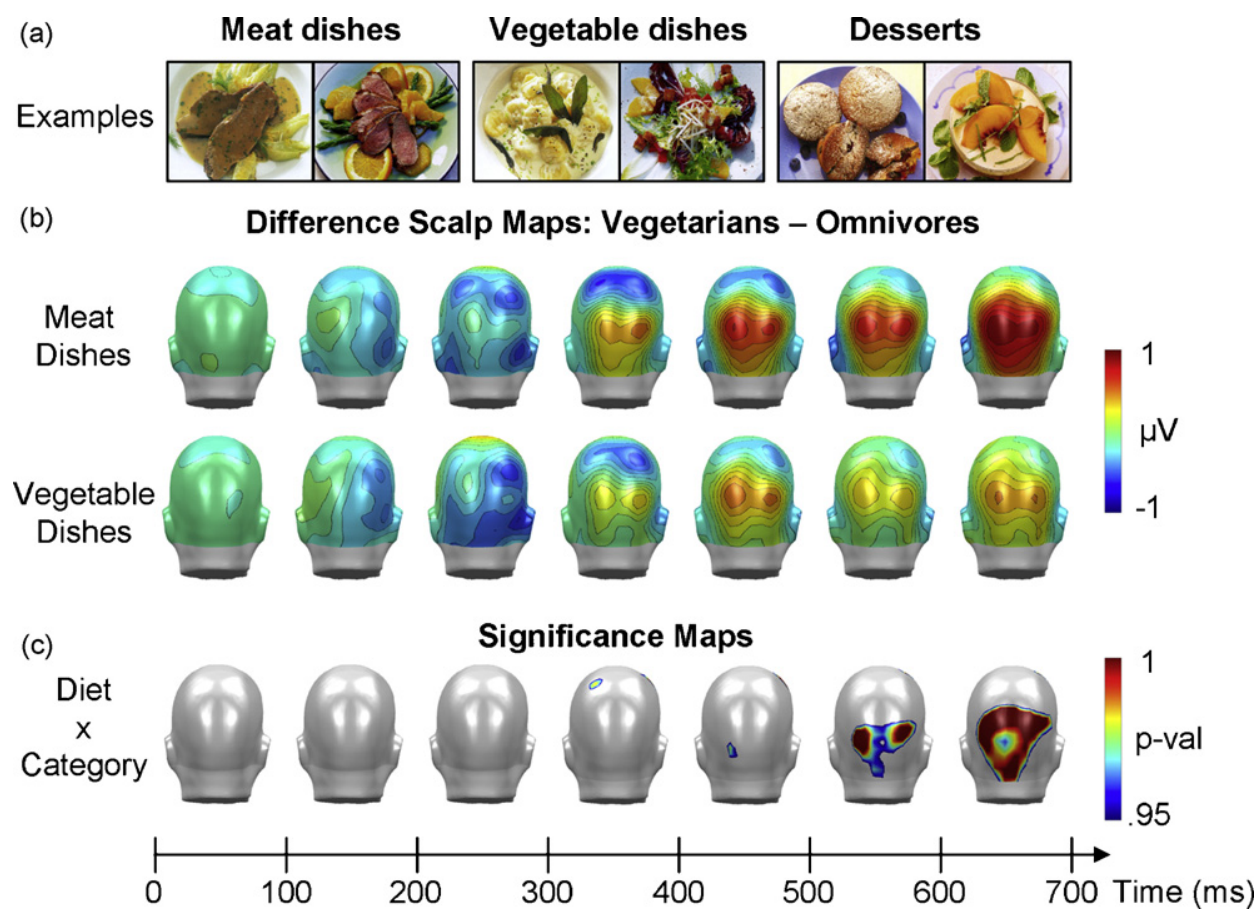

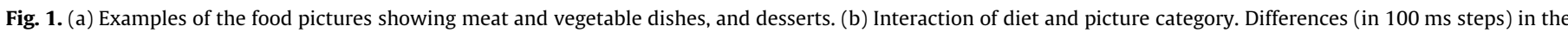

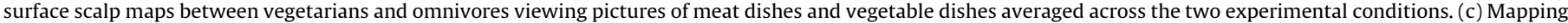
of the areas showing a significant interaction (back view of a model head).

\section{Methods}

\section{Participants}

Based on their eating habits, 24 participants ( 12 females; mean age $=24$, range 1931 ) were selected out of 38 students from the University of Greifswald. Participants who reported that they do not consume any meat products were classified as vegetarians $(N=12 ; 5$ females) and participants reporting no constraints with respect to the consumption of meat were classified as omnivores $(N=12 ; 7$ females). The mean body mass index was $22.4($ S.D. $=3.0)$ and did not differ between groups.

Vegetarianism can be related to restrictive forms of eating disorders (Bas, Karabudak, \& Kiziltan, 2005; Martins, Pliner, \& O'Connor, 1999) or a desire for thinness, especially in young women (Worsley \& Skrzypiec, 1998). Accordingly, screening questions related to eating disorders (anorexia, bulimia) and binge eating periods from the diagnostic interview for mental disorders (DIPS; Margraf, Schneider, \& Ehlers, 1994) served to exclude volunteers with eating disorders from participation. Furthermore, the German adaptation of the Restraint Scale (FEV) served to exclude restrained eaters (Heatherton, Herman, Polivy, King, \& McGree, 1988; Pudel \& Westenhöfer, 1989). In the final sample, vegetarians $(M=2.5, S . D .=1.6)$ and omnivores $(M=2.8$, $S . D .=2.1)$ scored equally low on the FEV Restrained scale (range: 0 21).

Vegetarians were asked to report how, when, and why they came to avoid meat (Rozin et al., 1997). They had followed their diet for an average of 8 years (range: 415 years) and the majority reported moral reasons for choosing a vegetarian diet, followed by disgust, taste and health motives.

All participants received 15 Euro for participation. In addition, they could earn 2.5 Euro depending on their performance in the explicit counting task.
Stimulus materials

Food pictures showed meat dishes $(N=100)$, vegetable dishes $(N=100)$ and desserts $(N=100)$ taken from commercially avail able cooking books (Fig. 1a). The meat and vegetable pictures showed principal meals and were matched with respect to amount, form, and colors of the displayed items. The dessert pictures showed cakes, fruits, and sweets.

\section{Procedure}

The 300 food pictures were presented in a pseudo randomized order with no more than two repetitions of the same category. Pictures were displayed for $1000 \mathrm{~ms}$ and variable inter trial intervals (1750 $2250 \mathrm{~ms}$ ). Each participant received his/her own order of picture presentation. In the first experimental condition, participants were instructed to passively view the pictures (passive viewing). This was followed by an active task condition in which participants saw the picture set again and were asked to silently count the dessert pictures (explicit task). At five interspersed breaks, they had to mention their count. Finally, they had to rate each of the depicted food on a 3 point hedonic rating scale with "do like it a lot" [1], "neither/nor" [0] or "do not like it at all" $[-1]$.

\section{EEG recording}

Brain and ocular scalp potential fields were measured with a 129 lead geodesic sensor net (EGI: Electrical Geodesics, Inc., Eugene, OR), on line bandpass filtered from 0.01 to $100 \mathrm{~Hz}$, and sampled at $250 \mathrm{~Hz}$ using Netstation software and EGI amplifiers. Electrode impedance was kept below $30 \mathrm{k} \Omega$, as recommended for this type of electroencephalogram (EEG) amplifier. Data were recorded continuously with the vertex sensor as reference. Data 
editing and artifact rejection were based on the statistical control of artifacts, specifically tailored for the analyses of dense sensor ERP recordings (Junghöfer, Elbert, Tucker, \& Rockstroh, 2000). The data were baseline corrected and converted to an average reference. Separate average waveforms were calculated for the six experimental cells (three food categories by two task conditions), and for each sensor and participant.

\section{Data analysis}

\section{Processing of food pictures}

For statistical analysis, single sensor waveform and mean area scoring were used in concert. In single sensor waveform analyses, repeated measure analyses of variance (ANOVAs) containing the within subjects factors Food Category (meat vs. vegetable dishes) and Task (passive viewing vs. explicit task) and the between subjects factor Diet Group (vegetarians vs. omnivores) were calculated for each time point after picture onset separately for each individual sensor (Schupp, Junghöfer, Weike, \& Hamm, 2003). To effectively prevent spurious findings, significant effects were only considered meaningful, when the effects were observed for at least eight continuous data points $(32 \mathrm{~ms})$ and two neighboring sensors (cf., Sabbagh \& Taylor, 2000). Results revealed a significant interaction of Food Category and Diet Group in the time interval from 500 to $700 \mathrm{~ms}$ over posterior sensor sites. ${ }^{1}$ To further explore these effects, mean amplitudes from representative posterior sensor clusters identified by both visual inspection and waveform analyses were averaged (EGI sensor numbers of the left cluster: 59 , $60,65,66,67,71,72$; right cluster: $77,78,84,85,86,91,92)$. As initial data analyses revealed similar effects for left and right sensor groups, the factor Laterality was not further considered in the main analyses for brevity of result presentation. Data were entered into a three factorial ANOVA including the factors Diet Group, Food Category, and Task.

\section{Explicit attention task}

The primary aim of the counting task was to examine the hypothesis that increased attention to meat pictures in vegetarians reflects an implicit process. A possible concern is that the experimental groups differ in their task performance or compliance regarding the explicit attention task. To assure comparable task performance in vegetarians and omnivores, a first analysis examined the counting task. An overall accuracy score was obtained by subtracting the sum of the deviations from the correct target count across the five times participants indicated their count, converted to a percentage score. Vegetarians and omnivores showed a high and comparable performance in the counting task with $M=97.1 \%$ (S.D. $=2.0)$ and $M=97.7 \%$ accuracy (S.D. $=1.4)$, respectively, $t(22)<1$, ns. In addition, dessert picture processing is associated in the explicit task condition with a pronounced target P3 in the ERP waveform (Johnson, 1988). Accordingly, in a further control analysis, the P3 to dessert pictures was scored as mean ERP activity from 400 to $600 \mathrm{~ms}$ in a centro parietal sensor cluster and submitted to a repeated measures ANOVA containing the between factor Diet Group and the within factor Task (passive viewing vs. explicit task). Participants showed the target P3 effect of enhanced P3 amplitudes in the explicit compared to the passive viewing task condition (Task $\left.F_{1,22}=91.5, p<0.0001\right)$. Of most interest, this effect was similarly pronounced in both groups (Task $\times$ Diet Group $F_{1,22}<1$ ).

\footnotetext{
${ }^{1}$ In this analysis, dessert pictures were excluded because of their different status in passive viewing and the explicit attention task. Control analysis including all three food categories confirmed a significant interaction of Food Category $\times$ Diet Group, $F_{2,44}=3.6 ; p<0.5$ and the absence of group differences for dessert pictures, $F_{1,22}=1.8 ;$ ns.
}

\section{Results}

\section{Stimulus ratings}

Vegetarians almost exclusively evaluated meat dishes as negative, resulting in highly significant different hedonic ratings between vegetarians and omnivores, $\chi^{2}(2,2400)=1293.9$, $p<0.001$. Compared to the prominent difference in evaluating meat dishes, only small albeit statistically significant group differences were found regarding vegetable dishes and desserts. Vegetarians rated vegetable dishes, $\chi^{2}(2,2400)=20.0, p<0.05$, and desserts, $\chi^{2}(2,2400)=100.0, p<0.05$, more positively than omnivores.

\section{Processing of food pictures: ERPs to meat vs. vegetables dishes}

As illustrated in Fig. 1b, meat pictures elicited enlarged LPPs over posterior regions in vegetarians compared to omnivores. In contrast, group differences of LPPs were small when processing vegetable dishes. These findings were supported by single sensor waveform analyses revealing a significant interaction of Food Category and Diet Group in the time window from 500 to $700 \mathrm{~ms}$ over parietal and occipital sensor regions (Fig. 1c). In addition, ANOVA analyses of mean areas scores of left and right posterior sensor clusters supported the hypothesis of selective meat processing in vegetarians; Food Category $\times$ Diet Group: $F_{1,22}=7.1, p<0.05$. Contrasting both diet groups, meat pictures elicited a significantly enlarged LPP in vegetarians compared to omnivores $\left(F_{1,22}=5.3, p<0.05\right)$, while LPP amplitudes to vege table dishes were not significantly different between vegetarians and omnivores $\left(F_{1,22}=1.4\right.$, ns). Considering each diet group separately, vegetarians showed a significantly enlarged LPP to meat $(M=1.26 \mu \mathrm{V})$ compared to vegetable dishes $(M=0.74 \mu \mathrm{V})$ $\left(F_{1,11}=13.4, p<0.005\right)$. In contrast, omnivores revealed similar LPP amplitudes to meat $(M=0.30 \mu \mathrm{V})$ and vegetable dishes $(M=0.24)\left(F_{1,11}=0.6, \mathrm{~ns}\right)$.

Remarkably, the interaction of Food Category and Diet Group was not further qualified by the factor Task (three way interac tion: $F_{1,22}=1.6, n s$ ). Separate examination of the passive viewing and explicit task condition confirmed enlarged LPP amplitudes in vegetarians viewing meat dishes compared to vegetable dishes in both task conditions $\left(\Delta=0.41\right.$ and $0.64 \mu \mathrm{V} ; F_{1,11}=13.2$ and 11.3, $p<0.005$, respectively) while no significant difference between meat and vegetable dishes was observed for omnivores ( $\Delta=0.15$ and $-0.01 \mu \mathrm{V} ; F s_{1,11}<1.5$, ns).

\section{Discussion}

Vegetarians evince a hedonic shift towards meat stimuli based on ideational reasoning, predominantly driven by moral and health concerns (Rozin et al., 1997). The present study explored the hypothesis that increased salience of meat in vegetarians turns corresponding food stimuli into efficient attention catchers. The LPP as non obtrusive brain potential component was measured, which has been shown to reveal a heightened state of selective attention when viewing affective pictures (Nieuwenhuis, Aston Jones, \& Cohen, 2005; Schupp et al., 2006). Consistent with the hypothesis, meat pictures elicited enlarged LPP amplitudes in vegetarians compared to omnivores. This finding was specific to meat stimuli as brain potentials to comparable pictures of vegetable dishes did not differ significantly between vegetarians and omnivores. These findings support the idea that the attention capture of food is not only related to biological processes of food consumption but also shaped by the affective salience associated with ideational reasoning and symbolic meaning (Rozin, 1996). 
The explicit attention task condition provides further insight into the nature of the attention capture of affect laden food cues. While participants were asked to count the 'desserts', meat as well as non meat principle meals were non target stimuli. Consistent with the assumption that the attention capture of meat stimuli in vegetarians reflects an obligatory process, the effect of enlarged LPP amplitudes to meat stimuli in vegetarians was fully main tained in the explicit task condition. Moreover, as behavioral and electrophysiological indices (target P3 effect) were highly similar across groups, this result rules out different task engagement as alternative explanation. Taken together, these findings suggest that the attention capture of meat pictures in vegetarians is due to their implicit stimulus relevance (Ito \& Cacioppo, 2000), which impacts the encoding even if the focus of explicit attention is directed to a different stimulus category.

Providing first evidence from the food domain, these findings underscore the notion to consider information processing at the level of distinct processing stages such as stimulus evaluation and behavioral responding (Cacioppo, Berntson, \& Gardner, 1999; Luck, Woodman, \& Vogel, 2000). Meat pictures not only elicit a strong avoidance predisposition in vegetarians, as revealed by subjective self reports, they are also highly efficient in recruiting selective visual attention during encoding, as indexed by the LPP. In the case of vegetarianism, increased attention to meat may foster attitude consistent behaviors by pitting moral reasoning (e.g., treatment of animals or long term health risks) higher than biological factors (flavor or nutritional value). The presentation of low and high caloric food items provides a further manipulation of the affective salience and symbolic meaning of food cues. Functional magnetic resonance imaging studies revealed reliable differences in the activation of perceptual and reward related brain regions when comparing high and low caloric food pictures (Killgore et al., 2003). These findings may have broader implications regarding the power of affect laden food cues to capture attentional resources. For instance, ambivalent and affect laden attitudes towards palatable food, common in restrained eating, dieting, and eating disorders, may increase implicit attention capture potentially opposing self control efforts (Mauler, Hamm, Weike, \& Tuschen Caffier, 2006).

\section{References}

Allen, M. W., Wilson, M., Ng, S. H., \& Dunne, M. (2000). Values and beliefs of vegetarians and omnivores. Journal of Social Psychology, 140, 405-422.

Amato, P., \& Partridge, S. (1989). The new vegetarians: promoting health and protecting life. New York, NY: Plenum Press.

Bas, M., Karabudak, E., \& Kiziltan, G. (2005). Vegetarianism and eating disorders: association between eating attitudes and other psychological factors among Turkish adolescents. Appetite, 44, 309-315.

Cacioppo, J. T., Berntson, G. G., \& Gardner, W. L. (1999). The affect system has parallel and integrative processing components: form follows function. Journal of Personality and Social Psychology, 76, 839-855.
Cuthbert, B. N., Schupp, H. T., Bradley, M. M., Birbaumer, N., \& Lang, P. J. (2000). Brain potentials in affective picture processing: covariation with autonomic arousal and affective report. Biological Psychology, 52, 95-111.

Fessler, D. M., Arguello, A. P., Mekdara, J. M., \& Macias, R. (2003). Disgust sensitivity and meat consumption: a test of an emotivist account of moral vegetarianism. Appetite, $41,31-41$.

Heatherton, T. F., Herman, C. P., Polivy, J., King, G. A., \& McGree, S. T. (1988). The (mis)measurement of restraint: an analysis of conceptual psychometric issues. Journal of Abnormal Psychology, 97, 19-28.

Ito, T. A., \& Cacioppo, J. T. (2000). Electrophysiological evidence of implicit and explicit categorization processes. Journal of Experimental Social Psychology, 36, 660-675.

Johnson, R. J. (1988). The amplitude of the P300 component of the event-related potential. Advances in Psychophysiology, 3, 69-137.

Junghöfer, M., Elbert, T., Tucker, D. M., \& Rockstroh, B. (2000). Statistical control of artifacts in dense array EEG/MEG studies. Psychophysiology, 37, 523-532.

Kenyon, P. M., \& Barker, M. E. (1998). Attitudes towards meat-eating in vegetarian and non-vegetarian teenage girls in England-an ethnographic approach. Appetite, 30(2), 185-198.

Killgore, W. D., Young, A. D., Femia, L. A., Bogorodzki, P., Rogowska, J., \& Yurgelun-Todd, D. A. (2003). Cortical and limbic activation during viewing of high- versus lowcalorie foods. NeuroImage, 19(4), 1381-1394.

Lang, P. J., Bradley, M. M., \& Cuthbert, B. N. (1997). Motivated attention: affect, activation, and action. In P. J. Lang, R. F. Simons, \& M. Balaban (Eds.), Attention and emotion: sensory and motivational processes (pp. 97-135). Mahwah, NJ: Erlbaum.

Luck, S. J., Woodman, G. F., \& Vogel, E. K. (2000). Event-related potential studies of attention. Trends in Cognitive Sciences, 4, 432-440.

Mauler, B. I., Hamm, A. O., Weike, A. I., \& Tuschen-Caffier, B. (2006). Affect regulation and food intake in bulimia nervosa: emotional responding to food cues after deprivation and subsequent eating. Journal of Abnormal Psychology, 115(3), 567579.

Margraf, J., Schneider, S., \& Ehlers, A. (1994). Diagnostisches Interview bei psychischen Störungen (DIPS; diagnostic interview for psychological disorders). Berlin: Springer

Martins, Y., Pliner, P., \& O'Connor, R. (1999). Restrained eating among vegetarians: does a vegetarian eating style mask concerns about weight? Appetite, 32(1), 145154.

Martins, Y., \& Pliner, P. (2005). Human food choices: an examination of the factors underlying acceptance/rejection of novel and familiar animal and nonanimal foods. Appetite, 45, 214-224.

Nieuwenhuis, S., Aston-Jones, G., \& Cohen, J. D. (2005). Decision making, the P3, and the locus coeruleus-norepinephrine system. Psychological Bulletin, 131, 510-532.

Öhman, A., Flykt, A., \& Lundqvist, D. (2000). Unconscious emotion: evolutionary perspectives, psychophysiological data and neuropsychological mechanisms. In R. D. Lane \& L. Nadel (Eds.), Cognitive neuroscience of emotion (pp. 296-327). New York: Oxford University Press.

Pudel, V., \& Westenhöfer, J. (1989). Der Fragebogen zum Essverhalten (FEV). Göttingen: Hogrefe.

Rozin, P., \& Vollmecke, T. A. (1986). Food likes and dislikes. Annual Review of Nutrition, 6, 433-456.

Rozin, P. (1996). Towards a psychology of food and eating: from motivation to module to model to marker, morality, meaning, and metaphor. Current Directions in Psychological Science, 5, 18-24.

Rozin, P., Markwith, M., \& Stoess, C. (1997). Moralization and becoming a vegetarian: the transformation of preferences into values and the recruitment of disgust. Psychological Science, 8, 67-73.

Sabbagh, M. A., \& Taylor, M. (2000). Neural correlates of theory-of-mind reasoning: an event-related potential study. Psychological Science, 11(1), 46-50.

Schupp, H. T., Junghöfer, M., Weike, A. I., \& Hamm, A. O. (2003). Emotional facilitation of sensory processing in the visual cortex. Psychological Science, 14, 7-13.

Schupp, H. T., Flaisch, T., Stockburger, J., \& Junghöfer, M. (2006). Emotion and attention: event-related brain potential studies. Progress in Brain Research, 156, 31-51.

Worsley, A., \& Skrzypiec, G. (1998). Teenage vegetarianism: prevalence, social and cognitive contexts. Appetite, 30(2), 151-170. 